Revista de Metalurgia 51(1)

Enero-Marzo 2015, e039

ISSN-L: 0034-8570

doi: http://dx.doi.org/10.3989/revmetalm.039

\title{
Aplicación de la técnica de ruido electroquímico al estudio de pinturas comerciales de efecto barrera
}

\author{
Santiago Lanchas García-Zarco ${ }^{\mathrm{a}}$, Víctor Alfonsín Pérez ${ }^{\mathrm{b}}$, Andrés Suarez García ${ }^{\mathrm{b}}$, \\ Santiago Urréjola Madriñán ${ }^{\mathrm{b}, \varpi}$, Ángel Sánchez Bermúdez ${ }^{\mathrm{a}}$

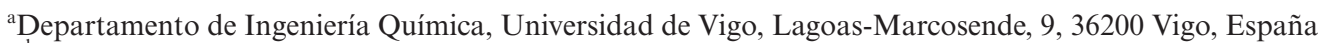 \\ ${ }^{b}$ Centro Universitario de la Defensa, Escuela Naval Militar, Plaza de España s/n 36920 Marín, España \\ Autor para la correspondencia: Santiago Urréjola: urrejola@cud.uvigo.es
}

Enviado: 21 Febrero 2014; Aceptado: 29 Enero 2015; Publicado on-Line: 10 Marzo 2015

RESUMEN: La medida e interpretación del Ruido Electroquímico (REQ) es uno de los métodos de análisis utilizados para la interpretación del fenómeno de la corrosión. Presenta, frente a otros tipos de metodologías, una serie de ventajas como son su sencillez, bajo coste y el hecho de ser un método no perturbativo. Esta metodología se ha mostrado eficaz en el estudio de sistemas metal desnudo-electrolito combinada con otras técnicas de análisis. En este caso se aplicó al estudio de pinturas comerciales utilizadas en procesos de prevención de la corrosión de las que no se tenían otro tipo de información. Como principal resultado de este estudio se muestra la eficacia de la aplicación de la resistencia de ruido, ya verificada en ensayos a nivel de laboratorio, para explicar el comportamiento de sistemas de pinturas que actualmente se están utilizando en la industria.

PALABRAS CLAVE: Corrosión; Densidad de potencia espectral; Pinturas; Resistencia de ruido; Ruido electroquímico

Citation / Cómo citar este artículo: Lanchas García-Zarco, S., Alfonsín Pérez, V., Suarez García, A., Urréjola Madriñán, S., Sánchez Bermúdez, A. (2015) "Aplicación de la técnica de ruido electroquímico al estudio de pinturas comerciales de efecto barrera". Rev. Metal. 51(1): e039. doi: http://dx.doi.org/10.3989/revmetalm.039.

\begin{abstract}
Noise resistance applied to the study paints. Electrochemical noise is one of the methods of analysis used to interpret the phenomenon of corrosion. It has a number of advantages over other methodology types including its simplicity, its low cost and the fact that it does not disturb the system. This methodology appears to be effective together with other techniques in metal-electrolyte systems. In this case the technique is applied on its own on commercial anti-corrosion paints for which no information is available from other techniques. The main result of this study reveals the effectiveness of the noise resistance parameter, which had already been tested in the lab, when it is used to explain how the paint system behaves in industry.
\end{abstract}

KEYWORDS: Corrosion; Electrochemical noise; Noise resistance; Paints; Power spectral density

\section{INTRODUCCIÓN}

Según la norma ASTM D16-12 (ASTM International, 2012) una pintura es, "Una mezcla líquida heterogénea que se convierte en una película continua, sólida y opaca después de su aplicación" (ASTM International, 2012). Toda pintura, bien se trate de una imprimación, de una capa intermedia o de un esmalte o acabado, se compone fundamentalmente de tres partes principales, vehículo, pigmentos y aditivos (Olaya-Flórez y TorresLuque, 2012). El vehículo fijo y el pigmento son los componentes que permanecen en la película de pintura una vez seca, y el vehículo volátil, utilizado exclusivamente para hacer posible la aplicación de la pintura, se pierde totalmente por evaporación durante el secado. Las distintas composiciones y combinaciones de vehículo, pigmentos y aditivos 
son las responsables de que la respuesta frente a la corrosión sea diferente en cada caso.

Existen distintos métodos de estudio del comportamiento de sistemas metal pintura frente a la corrosión. Los métodos no perturbativos como la Espectroscopía de Ruido Electroquímico o simplemente Ruido Electroquímico (REQ) se están aplicando en la actualidad en todo tipo de estudios relacionados con los procesos de corrosión (Sarmiento et al., 2009). Los estudios de REQ se refieren a las fluctuaciones espontáneas de potencial y/o corriente que se producen en un electrodo que se corroe libremente. Parece razonable suponer, aún sin estar del todo claro, que el origen y mecanismo que rigen los procesos que lo generan son la manifestación de la superposición de los procesos anódico y catódico que coexisten en la interfase electrodo disolución. Las pequeñas desviaciones que este equilibrio dinámico presenta son recogidas por los instrumentos de medida constituyendo los registros de ruido electroquímico que, con el tratamiento matemático adecuado, darán información sobre el estado del sistema sometido a estudio (Gaona-Tiburcio et al., 2014).

En este trabajo se aplica esta técnica a pinturas comerciales cuyo mecanismo de protección frente a la corrosión es de efecto barrera únicamente y se comprueba cómo, según que parámetros se utilicen, esta técnica permite distinguir las distintas etapas por las que pasan los sistemas.

\section{MATERIALES Y MÉTODOS}

\subsection{Preparación de muestras}

Para la elaboración de las probetas utilizadas, se eligieron cuatro tipos diferentes de recubrimientos de uso habitual en la industria, debido a sus propiedades de protección frente a la corrosión, con los cuales se pintó el mismo sustrato metálico, un acero de bajo contenido en carbono $(0,096 \%)$ aleación AP02, el cual se suministra en forma de paneles normalizados de $0,8 \mathrm{~mm}$ de espesor, $75 \mathrm{~mm}$ de ancho y $153 \mathrm{~mm}$ de longitud. La elección de las mismas fue acordada con el fabricante dado que se pretendía trabajar con pinturas que, en el momento de la realización de este trabajo, se aplicaban habitualmente en la industria y la idea era aplicar en solitario la técnica de REQ a pinturas comerciales. Los cuatro sistemas elegidos fueron pinturas de acabado con características diferenciadas, cuyos componentes (expresados en $\%$ en peso) se indican a continuación:

- Sistema 01: esmalte de acabado alquídico, de color rojo, compuesto por un $35,73 \%$ de resina alquídica media en aceite; 5,70\% de pigmento colorante; $1,15 \%$ de aditivos y un $57,42 \%$ de disolventes.
- Sistema 02: esmalte acrílico-poliéster, de color rojo, compuesto por tres resinas: acrílica diluida al $40 \%(32,32 \%)$; alquídica diluida al $40 \%$ $(23,70 \%)$ y una tercera de poliéster diluida al $40 \%(8,62 \%)$. El resto de los componentes son un pigmento colorante $(4,87 \%)$ y los aditivos $(3,92 \%)$.

- Sistema 03: pintura de acabado oleorresinoso color blanco compuesta por un $34,40 \%$ de resina fenólica modificada; $12,00 \%$ de aceite secante modificado; $27,70 \%$ de pigmento de $\mathrm{TiO}_{2} ; 3,20 \%$ de aditivos y $22,70 \%$ de disolventes.

- Sistema 04: pintura de acabado clorocaucho, color gris, compuesta por un $36,30 \%$ de resina clorocaucho $10 \mathrm{cp} ; 24,4 \%$ de pigmento; $6,90 \%$ de aditivos y $32,4 \%$ de disolventes.

El proceso de preparación y aplicación de las pinturas se realizó de acuerdo a las especificaciones dadas por los fabricantes. Para la preparación de la superficie, las probetas fueron desengrasadas con cloroetileno y se aplicaron con un sistema automático por arrastre, velocidad $2 \mathrm{~mm} \mathrm{~s}^{-1}$ y varilla de $75 \mu \mathrm{m}$ en húmedo. Las pinturas curaron por evaporación del disolvente y reacción química con el oxígeno del aire y las probetas se utilizaron una semana después de haber alcanzado el curado "seco duro" todo ello de acuerdo a las fichas técnicas del fabricante.

Los espesores finales de cada probeta fueron 29,$6 ; 28,8 ; 33,9$ y $36,2 \mu \mathrm{m}$ para los sistemas 01 , 02,03 y 04 respectivamente.

\subsection{Toma de datos}

Para evitar que los registros de ruido electroquímico fuesen alterados por alguna influencia externa se utilizó una caja de Faraday en la que se colocó una celda electroquímica que contenía una disolución de $\mathrm{NaCl}$ 0,5 M la cual llevó en sus laterales ensamblados dos electrodos de trabajo idénticos, conectados a un amperímetro de resistencia cero, multímetro modelo Hewlett Packcard 3478, entre los que se midió la intensidad de corriente. La variación de la diferencia de potencial se registró por medio de un voltímetro modelo Solatrom 7060 Systems Voltmeter, entre uno de los electrodos de trabajo y un electrodo de referencia de calomelanos saturado, ECS. Tanto el voltímetro como el amperímetro fueron controlados por un ordenador al cual son enviados los datos obtenidos en los registros de ruido a través de un bus estándar IEEE488. En la Figura 1 se puede observar un esquema del equipo de medida (Espada et al., 2001; Homborg et al., 2012).

Los equipos se controlaron por medio de un ordenador, provisto de un programa especial para la adquisición de datos de ruido electroquímico, tanto de potencial como de intensidad. La frecuencia de muestreo empleada fue de $2 \mathrm{~Hz}$, lo que equivale a 


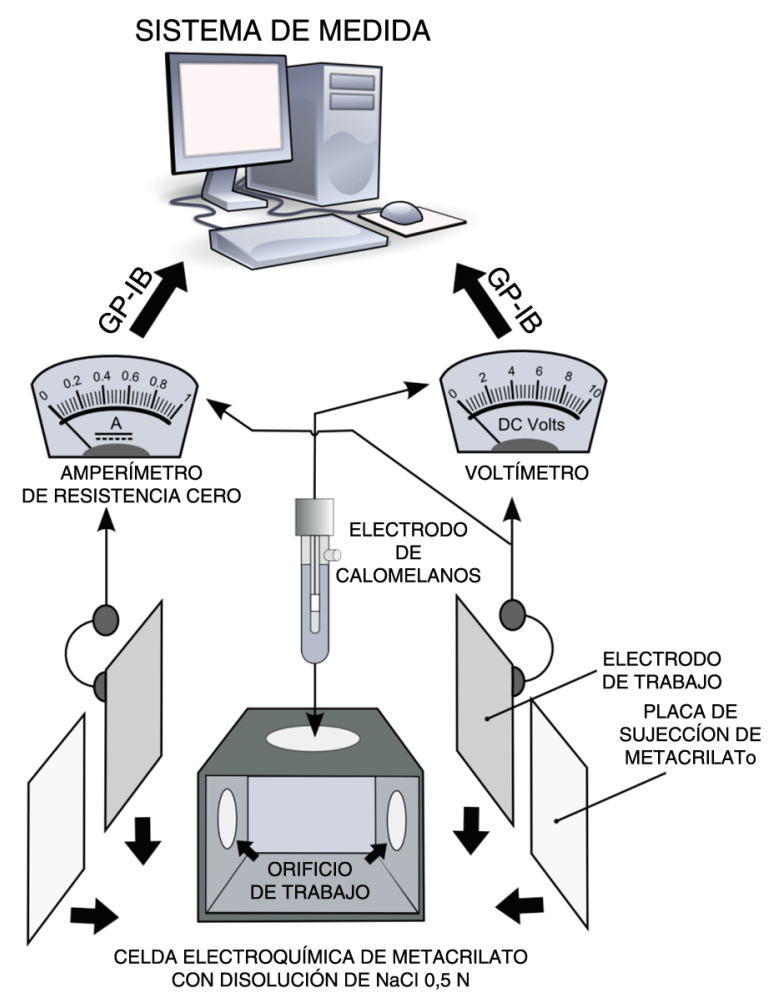

Figura 1. Esquema de medida.

dos puntos por segundo $(\Delta \mathrm{t}=0,5 \mathrm{~s})$. Cada registro de datos de potencial e intensidad de corriente se extendió a 2048 puntos. Los registros se realizaron hasta que se produjo la degradación de las pinturas. En cada uno de ellos se calcularon los valores medios de los parámetros indicados en el apartado siguiente, a partir de los cuales, se construyeron las gráficas potencial $v s$. tiempo que aparecen en este trabajo, y que permiten comprobar la degradación de las pinturas a lo largo del tiempo.

El primer registro puntual de tiempo, se realizó en el momento de la inmersión y el último, una vez aparecieron los productos de corrosión, teniendo las probetas sometidas al electrolito hasta ese momento. Durante todo este proceso se realizaron exámenes visuales periódicos para determinar la aparición de ampollamiento, puntos de corrosión o cualquier otro signo de degradación en la superficie de la capa de pintura.

\subsection{Tratamiento de datos}

Una vez que se obtuvieron los diferentes registros de ruido se procedió al tratamiento matemático de los mismos. Este tratamiento se llevó a cabo en el dominio del tiempo, por medio de análisis estadístico, y en el dominio de la frecuencia, mediante el análisis de Fourier. Los parámetros calculados en cada registro fueron los valores medios de potencial $(V)$ e intensidad (I); desviaciones estándar de potencial $(\sigma \mathrm{V})$ e intensidad $(\sigma \mathrm{I})$; resistencia de ruido $(\mathrm{Rn}=\sigma \mathrm{V} / \sigma \mathrm{I})$; densidad de potencia espectral de potencial [DPE (V)] y la intensidad [DPE (I)].

El tratamiento estadístico se llevó a cabo normalizando los valores de potencial según la ecuación (1):

$V_{N}=\frac{V-V_{m}}{\sigma V}$

Siendo $\mathrm{V}_{\mathrm{N}}$ los valores del potencial normalizado; $\mathrm{V}$ el potencial sin normalizar; $\mathrm{V}_{\mathrm{m}}$ el valor medio del potencial y $\sigma \mathrm{V}$, la desviación estándar del potencial.

El cálculo de las desviaciones estándar de los registros de potencial e intensidad permitió calcular la resistencia de ruido $\left(R_{r}\right)$ como el cociente entre las desviaciones estándar de los registros de potencial e intensidad, de acuerdo con la ecuación (2):

$\mathrm{R}_{\mathrm{r}}=[\sigma \mathrm{V}] /[\sigma \mathrm{I}]$

El cociente $R_{r}$, es uno de los parámetros más utilizados para el seguimiento de la evolución de sistemas electroquímicos estudiados mediante la técnica de ruido electroquímico, como es el caso de este estudio de sistemas metal-electrolito (Deyá et al., 2013).

En el caso del análisis de Fourier el parámetro elegido fue la densidad de potencia espectral (DPE), cuyo valor se puede calcular por distintos métodos (Pujar et al., 2007), basados tanto en la transformada rápida de Fourier (TRF) como en el método de la máxima entropía (MEM) dando resultados similares todos ellos. En este caso el método de cálculo elegido fue el cuadrado de la transformada de Fourier (en decibelios) frente a la frecuencia, en un gráfico logarítmico. Se han normalizado los registros de potencial en la media aritmética, con el fin de eliminar así la contribución de la intensidad de corriente continua debida a la medida del potencial frente al electrodo de referencia. Para la realización de las representaciones gráficas se importaron los valores de los registros de ruido, así como los calculados a través del programa MATLAB ${ }^{\circledR}$, a una hoja de cálculo de Microsoft ${ }^{\circledR}$ Excel.

\section{RESULTADOS}

La evolución del potencial en estos sistemas permitió distinguir cuatro etapas en la vida de los mismos (Faidi et al., 1993): una etapa inicial (EI) en la cual el electrolito va empapando paulatinamente la pintura; una segunda fase denominada etapa de efecto barrera (EEB), en la cual la pintura protege totalmente al metal de la corrosión hasta que este efecto empieza a desaparecer dando paso a la siguiente etapa, denominada pérdida de efecto barrera (PEB) durante la cual la pintura continúa 
degradándose paulatinamente, dando lugar a una etapa final $(\mathrm{EF})$, en la cual el efecto de protección de la pintura se ha perdido totalmente.

En la interpretación de las gráficas de variación del potencial medio con el tiempo hay que tener en cuenta el hecho de que los sistemas estudiados tienen diferente tiempo de respuesta frente a la corrosión. En las Figuras 2 y 3 se muestran estas etapas para los sistemas 1 y 2 , donde se puede apreciar cómo a medida que el recubrimiento se va degradando, el potencial se acerca hacia un valor en torno a los $-0,680 \mathrm{~V}$, y que es el potencial medido en el sistema metal desnudo-electrolito. El mecanismo de protección de las pinturas ensayadas se produce fundamentalmente por efecto barrera y se pueden destacar dos patrones de comportamiento distintos.
Primero: las pinturas 01 y 03 en las cuales los potenciales de corrosión se mantienen en valores de potencial menos negativos durante las etapa de efecto barrera $(-0,15 \mathrm{a}-0,28 \mathrm{~V})$ y apenas son más negativos de $-0,4$ cuando van perdiendo esta protección. En estas pinturas el mecanismo de formación de película se realiza por medio de una reacción química entre la pintura con el oxígeno del aire. Son pinturas con pobre resistencia química, tienden a saponificarse en medios alcalinos, teniendo también una pobre resistencia al agua.

Segundo: las pinturas 02 y 04 en las cuales los potenciales de corrosión ya son más negativos que $-0,4$ durante la etapa de efecto barrera, superando los $-0,5 \mathrm{~V}$ en la etapa que van perdiendo su efecto barrera. En estas pinturas el mecanismo de formación de película es por medio de la

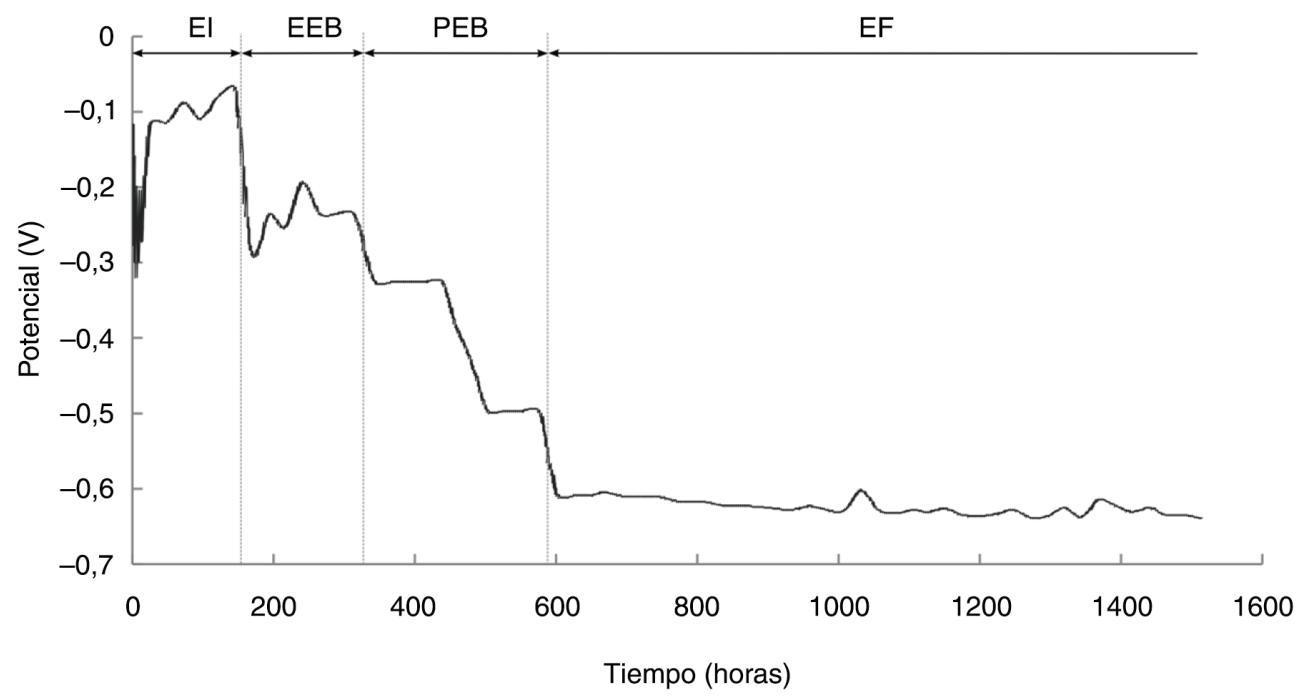

FiguRA 2. Variación del potencial medio. Sistema 01.

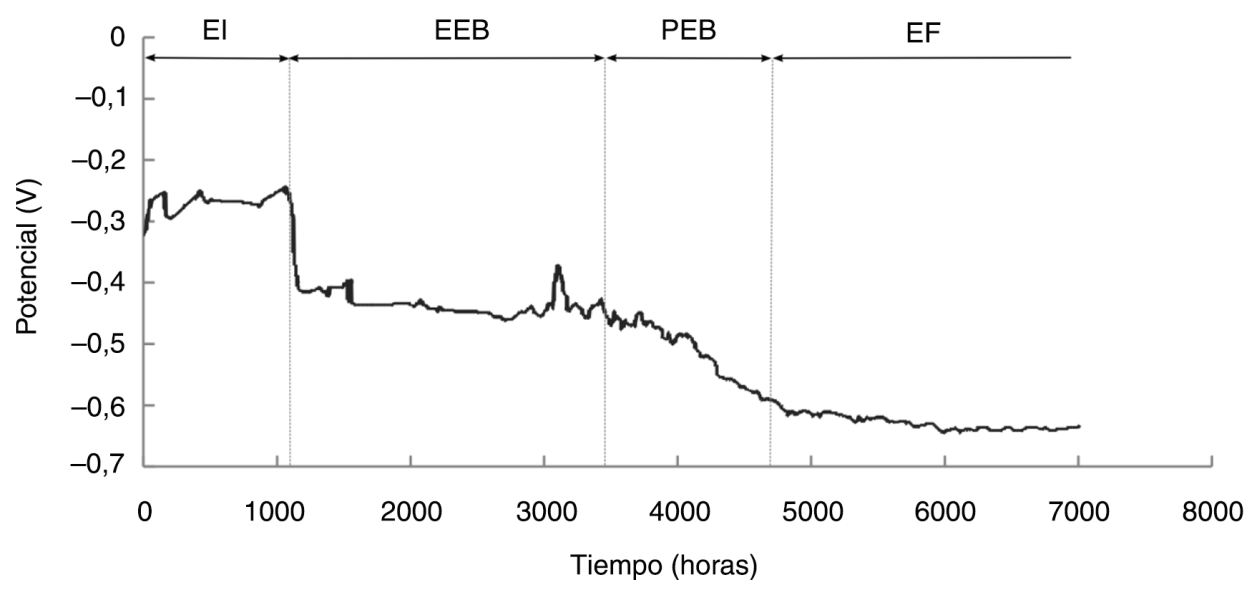

Figura 3. Variación del potencial medio. Sistema 02. 
evaporación del disolvente, por lo que tienen una buena resistencia química y buena resistencia al agua, lo que hace que el efecto de protección dure más tiempo (Hare, 1995).

Si se analizan los registros generados, en todos los sistemas tras un tránsito por el potencial de corrosión del hierro, $-0,660 \mathrm{~V}$ frente a ECS, el potencial sigue evolucionando hacia valores más cercanos al obtenido en el caso de las medidas con metal desnudo, a medida que se depositan sobre él productos de corrosión.

\section{DISCUSIÓN}

En el presente estudio se pudo corroborar la existencia de estas etapas, siendo la duración de cada uno de ellas distinta, dependiendo del sistema sometido a estudio. Se observó una diferencia de duración de las etapas entre el sistemas 01 (577 horas hasta el inicio de la etapa final) y los sistemas 02 y 04 (en torno a las 4700 horas de duración hasta el inicio de la etapa final), la cual puede ser explicada por los distintos mecanismos de formación de película que utilizan las cuatro pinturas estudiadas. El caso anómalo de la alta duración de la pintura 03 pudiera ser explicado por el alto contenido $(27,70 \%)$ en pigmento de bióxido de titanio $\left(\mathrm{TiO}_{2}\right)$, y que hace que pese a tener el mismo mecanismo de formación de película que la pintura 01, su tiempo de vida se aproxime, e incluso supere, 5153 horas a los sistemas 02 y 04 frente a las otras pinturas que no llevan este tipo de pigmento. La presencia de dicho pigmento hace mejorar las propiedades anticorrosión de las mismas (Ahmed y Selim, 2010). La aplicación de la técnica de ruido electroquímico sólo permite detectar esta anomalía pero no nos permite explicarla. Un estudio con otras técnicas sería conveniente para profundizar en este hecho, pero va más allá a los objetivos de este trabajo.

En las Figuras 2 y 3, en las que se representan la variación del potencial medio frente al tiempo (sistemas 1 y 2), se pueden observar los distintos periodos de evolución mencionados anteriormente. La variación de la intensidad durante todo el periodo experimental (unas horas) es pequeña, al igual que en el caso del metal sin recubrir, aportando escasa información sobre el comportamiento de las pinturas.

\subsection{Evolución de las pinturas}

Como se dijo anteriormente la evolución del potencial medio permite distinguir cuatro etapas en la evolución de las pinturas. Los registros de potencial en los cuatro sistemas estudiados, independientemente de la pintura, presentan tránsitos similares que solo dependen de la etapa en la que se encuentre el recubrimiento (Espada et al., 2001; Homborg, et al., 2012).

\subsubsection{Etapa inicial (EI)}

La etapa en la que el sistema se está empapando, suele tener una duración más corta en todos los sistemas. En esta etapa tanto la medida de la diferencia de potencial entre el metal y el electrodo de referencia, como la medida de intensidad entre los dos electrodos de trabajo, está dificultada por la resistencia de la pintura a ser impregnada por el electrolito. El electrolito va rellenando paulatinamente los huecos del recubrimiento y, a medida que alcanza la superficie del metal, se va produciendo un mejor contacto eléctrico, lo que hace que aparezcan saltos de potencial en un mismo registro desde valores muy negativos hasta valores positivos.

\subsubsection{Etapa de efecto barrera (EEB)}

Las oscilaciones en las gráficas potencial-tiempo confieren a las mismas un aspecto de ruido browniano en todos los sistemas con menos picos que en la etapa anterior, tal y como se aprecia en la Figura 4, apreciándose en esta etapa continuas ondulaciones en las representaciones potencial-tiempo.

Al final de la etapa, cuando el efecto barrera prácticamente ha desaparecido, y los productos de corrosión comienzan a aflorar a la superficie de la capa de pintura, se observa la aparición de un gran número de picos, dando lugar a la etapa siguiente.

\subsubsection{Pérdida del efecto barrera (PEB)}

Como era de esperar los registros de potencial empiezan siendo similares a los de la etapa anterior, lo cual dificulta en algunos casos su distinción. Este hecho se puede comprobar comparando las Figuras 4 y 5 . Por otro lado, lo más característico de esta etapa es que, al final de la misma, los últimos registros muestran ondulaciones de potencial cada vez más suaves, similares a las obtenidas para el metal desnudo. De esto se concluye que los perfiles de ruido adoptan una forma similar a los obtenidos en el metal base degradado por corrosión, correspondiente al final de la etapa de PEB. De todo ello se concluye que el análisis aislado de estos gráficos puede no ser suficiente para determinar la etapa de degradación en la que se encuentra la pintura.

\subsubsection{Etapa final ( $E F)$}

En la etapa final el potencial se mantiene constante en valores en torno a los $-0,700 \mathrm{~V}$. En esta etapa los productos de corrosión toman preponderancia adoptando las gráficas un perfil similar al obtenido en el acero sin recubrir, sin presentar picos apreciables con variaciones que pueden ser ondulantes y a veces escalonadas, muy diferentes a los de la etapa anterior tal y como se puede apreciar en la Figura 5. 


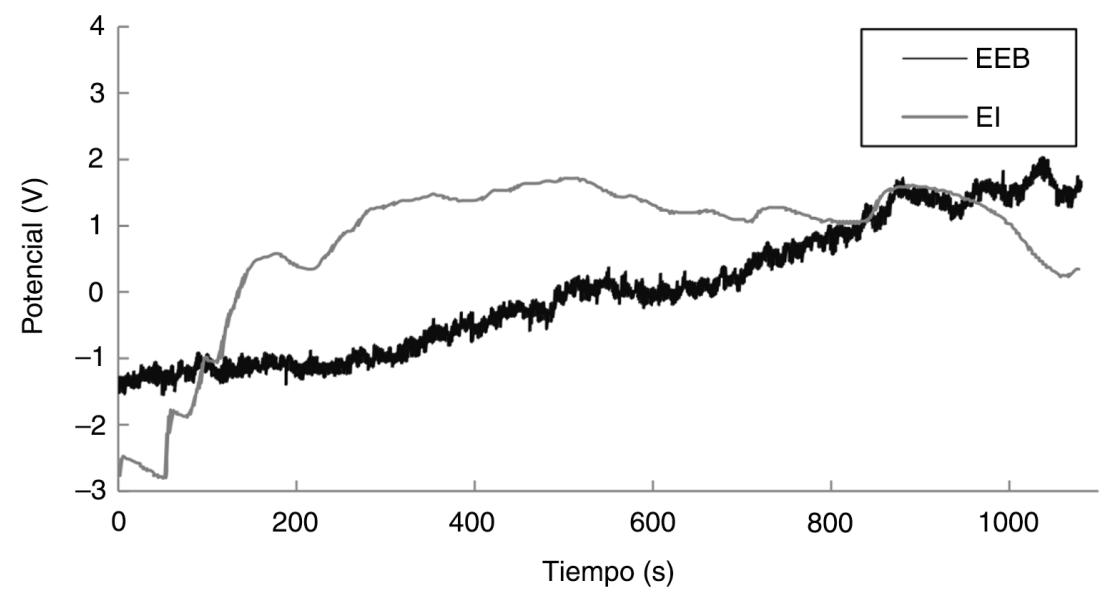

FIgURA 4. Comparación de registro de potencial entre la Etapa Inicial y la Etapa de Efecto Barrera. EI (50 horas de exposición Pintura 01), EEB (1514 horas de exposición Pintura 02).

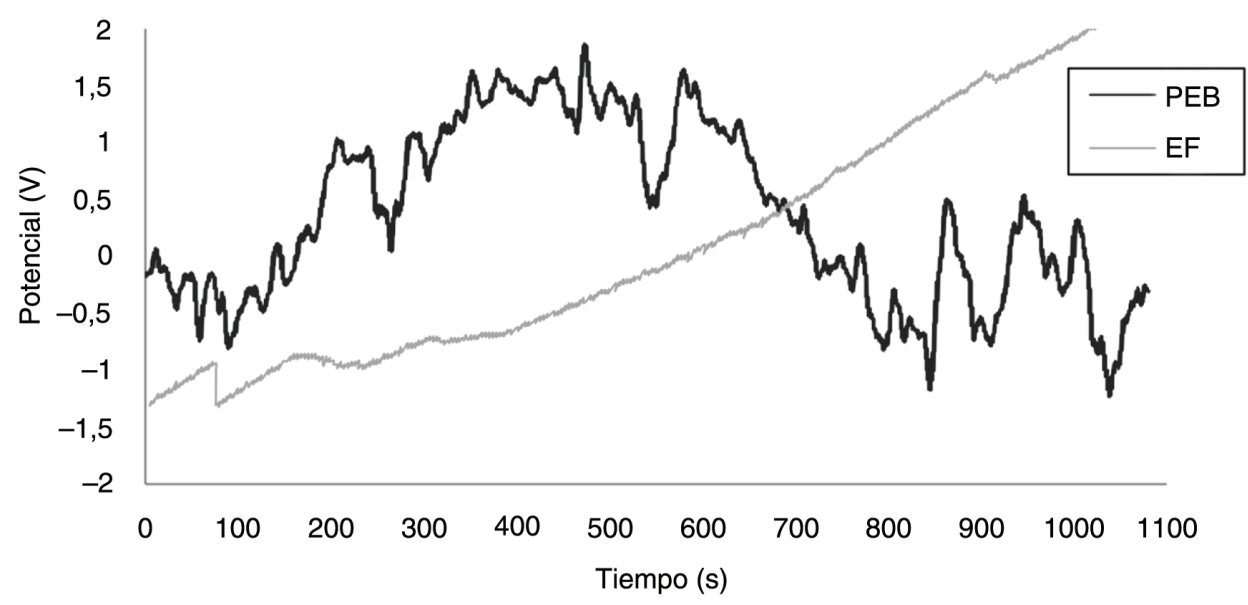

Figura 5. Comparación de registro de potencial entre la Etapa de Pérdida Efecto Barrera y la Etapa Final. PEB (3729 horas de exposición Pintura 03), EF (4822 horas de exposición Pintura 04).

\subsection{Análisis de los valores de resistencia de ruido}

Pese a la buena información que provee el estudio de los valores de potencial, ésta se complementa con el uso de la resistencia de ruido, un parámetro de larga tradición en los estudios de ruido electroquímico. Su uso es debido a que, como puede apreciarse en la Tabla 1, en distintas etapas de degradación de un recubrimiento se pueden observar valores similares de potencial, lo que no ocurre con las lecturas de los valores de la resistencia de ruido. Por ejemplo, los valores medios de potencial en el caso del sistema 01 son prácticamente idénticos en la etapa EI que en la etapa EEB, situándose entre los $-0,100 \mathrm{~V}$ y $-0,300 \mathrm{~V}$ en ambas etapas. Sin embargo, los valores de $\mathrm{R}_{\mathrm{r}}$ son notablemente distintos $\left(1,57 \cdot 10^{9} \Omega\right.$ en la etapa de EI y $1,06 \cdot 10^{8} \Omega$ en la etapa de EEB).

Tanto en los primeros estudios sobre ruido electroquímico (Mansfeld et al., 1997; Skerry y Eden,
1987), como en estudios más actuales (Shao et al., 2009; Deyá et al., 2013), valores altos de resistencia de ruido se corresponden con recubrimientos en buen estado, y valores bajos se corresponden con la presencia de un proceso de corrosión avanzado. Este hecho puede ser corroborado leyendo la Tabla 1 donde se comparan los distintos valores de resistencia de ruido de los sistemas estudiados en varios estadios de degradación, con los obtenidos para el metal desnudo. En todos los casos se observa que, a medida que el recubrimiento se va degradando, el valor de la resistencia de ruido desciende.

\subsection{Análisis de los valores de densidad de potencia espectral}

En la mayoría de los estudios de ruido electroquímico se menciona que la pendiente de la densidad de potencia espectral (DPE) de los registros de ruido 
TABLA 1. Variación del potencial y de la resistencia de ruido

\begin{tabular}{llrcc}
\hline Etapa & \multicolumn{1}{c}{ Sistema } & T (horas) & \multicolumn{1}{c}{$\mathbf{V}^{\dagger}(\mathbf{V})$} & $\mathbf{R}_{\mathbf{n}}^{\S}(\mathbf{\Omega})$ \\
\hline EI & Metal desnudo & 2 & $-6,55 \times 10^{-1}$ & $1,64 \times 10^{4}$ \\
& Sistema 01 & 145 & $-1,59 \times 10^{-1}$ & $1,57 \times 10^{9}$ \\
& Sistema 02 & 1114 & $-2,77 \times 10^{-1}$ & $2,81 \times 10^{7}$ \\
& Sistema 03 & 1220 & $-2,76 \times 10^{-1}$ & $1,29 \times 10^{9}$ \\
& Sistema 04 & 425 & $-1,56 \times 10^{-1}$ & $6,57 \times 10^{8}$ \\
EEB & Sistema 01 & 168 & $-2,39 \times 10^{-1}$ & $5,18 \times 10^{8}$ \\
& Sistema 02 & 2393 & $-4,37 \times 10^{-1}$ & $6,78 \times 10^{8}$ \\
& Sistema 03 & 2120 & $-3,11 \times 10^{-1}$ & $5,94 \times 10^{8}$ \\
& Sistema 04 & 2195 & $-4,47 \times 10^{-1}$ & $8,56 \times 10^{8}$ \\
PEB & Sistema 01 & 264 & $-4,03 \times 10^{-1}$ & $1,06 \times 10^{8}$ \\
& Sistema 02 & 1245 & $-5,15 \times 10^{-1}$ & $4,89 \times 10^{6}$ \\
& Sistema 03 & 1813 & $-3,99 \times 10^{-1}$ & $3,95 \times 10^{8}$ \\
& Sistema 04 & 2077 & $-5,20 \times 10^{-1}$ & $5,36 \times 10^{8}$ \\
EF & Metal desnudo & $>2$ & $-7,15 \times 10^{-1}$ & $8,57 \times 10^{2}$ \\
& Sistema 01 & 936 & $-6,25 \times 10^{-1}$ & $1,01 \times 10^{8}$ \\
& Sistema 02 & 2262 & $-6,28 \times 10^{-1}$ & $2,95 \times 10^{6}$ \\
& Sistema 03 & 986 & $-6,32 \times 10^{-1}$ & $2,07 \times 10^{8}$ \\
& Sistema 04 & 1058 & $-6,49 \times 10^{-1}$ & $3,14 \times 10^{8}$ \\
\hline
\end{tabular}

${ }^{\dagger}$ Valores medios de potencial.

${ }^{\S}$ Valores medios de la resistencia de ruido en cada etapa.

electroquímico de intensidad y potencial es un factor que suministra una información útil sobre el proceso que ocurre en los electrodos. A lo largo de los años, en diversos trabajos se menciona que la pendiente de la DPE de potencial está íntimamente relacionada con el tipo de corrosión (uniforme, picaduras, etc.), mientras que los registros de la pendiente de la DPE de intensidad se relacionan con la velocidad de corrosión, si bien esto último está menos admitido (Cottis, 2001; Castañeda et al., 2010; Muniandy et al., 2011). No obstante hay que dejar constancia de que la gran mayoría de los trabajos que se recogen en la bibliografía, relativos al empleo de la técnica de ruido electroquímico para evaluar el comportamiento frente a la corrosión de sistemas electroquímicos, se centran en el estudio de sistemas metal electrolito, encontrándose muy pocas publicaciones que versen sobre sistemas metal pintura.

Comparando los sistemas estudiados, se puede apreciar que el denominador común es el hecho de que en todos los casos el valor de la densidad de potencia espectral de la intensidad de corriente es prácticamente constante a lo largo de todo el periodo de exposición, al contrario de lo que nos dice la bibliografía (Muniandy et al., 2011). Por el contrario no ocurre lo mismo con los registros de potencial, en los que los valores de la pendiente de la representación de la densidad de potencia espectral de potencial no permanecen constantes a lo largo del tiempo, sino que presentan continuas fluctuaciones. En la Tabla 2
TABLA 2. Variación de la densidad de potencia espectral de potencial

\begin{tabular}{lcccc}
\hline Etapa & Sistema & Máximo & Mínimo & Media \\
\hline EI & 01 & $-18,50$ & $-63,70$ & $-43,90$ \\
& 02 & $-16,72$ & $-65,15$ & $-61,22$ \\
& 03 & $-11,14$ & $-64,87$ & $-29,73$ \\
\multirow{4}{*}{ EEB } & 04 & $-21,30$ & $-63,15$ & $-40,55$ \\
& 01 & $-52,50$ & $-61,10$ & $-58,00$ \\
& 02 & $-14,26$ & $-65,27$ & $-62,30$ \\
& 03 & $-17,09$ & $-65,02$ & $-46,05$ \\
PEB & 04 & $-17,21$ & $-65,03$ & $-35,38$ \\
& 01 & $-63,90$ & $-65,00$ & $-64,50$ \\
& 02 & $-14,90$ & $-65,29$ & $-62,93$ \\
& 03 & $-24,48$ & $-64,69$ & $-48,38$ \\
EF & 04 & $-20,04$ & $-65,03$ & $-39,04$ \\
& 01 & $-36,40$ & $-65,00$ & $-52,60$ \\
& 02 & $-20,64$ & $-65,35$ & $-63,69$ \\
& 03 & $-30,21$ & $-65,03$ & $-52,82$ \\
& 04 & $-26,01$ & $-65,03$ & $-39,29$ \\
& 03 & & &
\end{tabular}

se comparan los valores máximos, mínimos y medios, obtenidos en las distintas etapas, para los sistemas estudiados, de los registros de potencial. No existe un patrón claro de comportamiento que nos permita agrupar en los periodos de vida de la pintura; lo único que puede intuirse es el hecho de que los cambios entre cada una de las etapas de cada uno de los sistemas coinciden con cambios significativos de la pendiente de la densidad de potencia espectral.

\section{CONCLUSIONES}

Haciendo un resumen se pueden dividir las etapas de vida de nuestras pinturas en las siguientes:

- EI: Etapa inicial en la que el sistema se está impregnando de electrolito y suele ser de corta duración en todos los sistemas. Tanto los valores de $\mathrm{R}_{\mathrm{r}}$ como los de potencial son de difícil interpretación.

- EEB: Etapa de efecto barrera. Se observa un aumento en los valores de $R_{r}$ al aparecer un mecanismo de protección. Las oscilaciones en las gráficas potencial-tiempo confieren a las mismas un aspecto de ruido browniano en todos los sistemas.

- PEB: Pérdida del efecto barrera. La $\mathrm{R}_{\mathrm{r}}$ vuelve a disminuir en valor acercándose a los que aparecerán en la etapa final. El aspecto de los registros de potenciales es similar a la anterior etapa, pero los perfiles se van suavizando.

- EF: Etapa final en la que la $R_{r}$ se estabiliza alcanzando el valor mínimo, y el potencial se mantiene constante en valores próximos a los $-0,700 \mathrm{~V}$. En esta etapa los productos de 
corrosión hacen notar su efecto, adoptando las gráficas un perfil similar al obtenido en el acero sin recubrir, esto es, no presentan saltos grandes sino ondulaciones suaves y a veces escalonadas.

- En todos los casos se observa que, a medida que el recubrimiento se va degradando, el valor de la resistencia de ruido desciende. Esta conclusión coincide con las que se pueden extraer de diversos estudios anteriores relativos a otros tipos de recubrimientos (Skerry y Eden, 1987), en los cuales valores altos de la resistencia de ruido se corresponden con recubrimientos en buen estado, y valores bajos se relacionan con la presencia de un proceso de corrosión avanzado.

- Pese a que el uso de valores de potencial por separado nos da una idea cualitativa del estado del recubrimiento pudiendo distinguir las etapas por las que pasa el mismo, el uso de la medida de valores de resistencia de ruido da una idea de cómo se encuentra un recubrimiento en cada momento.

\section{REFERENCIAS}

Ahmed, N.M., Selim, M.M. (2010). Anticorrosive performance of titanium dioxide-talc hybrid pigments in alkyd paint formulations for protection of steel structures. AntiCorros. Methods Mater. 57 (3), 133-141. http://dx.doi.org/ $10.1108 / 00035591011040092$.

ASTM International (2012). Standard Terminology for Paint, Related Coatings, Materials, and Applications. (ASTM D1612-05). West Conshohocken, PA, 2005, USA. http:// dx.doi.org/10.1520/D0016-12.

Castañeda, I., Romero, M., Malo, J.M., Uruchurtu, J. (2010). Electrochemical noise of the erosion-corrosion of copper in relation with its hydrodynamic parameters. Rev. Metal. 46 (5), 446-457. http://dx.doi.org/10.3989/revmetalm.1002.

Cottis, R.A. (2001). Interpretation of electrochemical noise data. Corrosion 57 (3), 265-285. http://dx.doi.org/10.5006/ 1.3290350 .

Deyá, M.C., Del Amo, B., Spinelli, E., Romagnoli, R. (2013). The assessment of a smart anticorrosive coating by the electrochemical noise technique. Prog. Org. Coat. 76 (4), 525-532. http://dx.doi.org/10.1016/j.porgcoat.2012.09.014.

Espada Recarey, L., Sánchez Bermúdez, A., Urréjola Madriñán, S., Bouzada Alvela, F. (2001). Noise resistance applied to the study of zinc rich paints. Rev. Metal. 37 (1), 24-33. http://dx.doi.org/10.3989/revmetalm.2001.v37.i1.438.

Faidi, S.E., Scantlebury, J.D., Bullivant, P., Whittle, N.T., Savin, R. (1993). An electrochemical study of zinc-containing epoxy coatings on mild steel. Corros. Sci. 35 (5-8), 1319-1328. http://dx.doi.org/10.1016/0010-938X(93)90354-J.

Gaona-Tiburcio, C., Aguilar, L.M.R., Zambrano, P., Estupiñán López, F., Cabral, J.A., Nieves-Mendoza, D., CastilloGonzález, E., Almeraya-Calderón, F. (2014). Electrochemical noise analysis of nickel based superalloys in acid solutions. Int. J. Electrochem. Sci. 9 (2), 523-533.

Hare, C.H. (1995). Protective Coatings: Fundamentals of Chemistry and composition. Surf. Coat. Int. 78, pp. 1-14-289-231.

Homborg, A.M., Tinga, T., Zhang, X., van Westing, E.P.M., Oonincx, P.J., de Wit, J.H.W., Mol, J.M.C. (2012). Timefrequency methods for trend removal in electrochemical noise data. Electrochim. Acta 70, 199-209. http://dx.doi. org/10.1016/j.electacta.2012.03.062.

Mansfeld, F., Xiao, H., Han, L.T., Lee, C.C. (1997). Electrochemical impedance and noise data for polymer coated steel exposed at remote marine test sites. Prog. Org. Coat. 30 (1-2), 89-100. http://dx.doi.org/10.1016/S0300-9440(96)00675-3.

Muniandy, S.V., Chew, W.X., Kan, C.S. (2011). Multifractal modelling of electrochemical noise in corrosion of carbon steel. Corros. Sci. 53 (1), 188-200. http://dx.doi.org/ 10.1016/j.corsci.2010.09.005.

Olaya-Flórez, J., Torres-Luque, M.M. (2012). Corrosion resistance of organic coatings through electrochemical impedance spectroscopy. Ingeniería y Universidad 16 (1), 43-58.

Pujar, M.G., Anita, T., Shaikh, H., Dayal, R.K., Khatak, H.S. (2007). Analysis of electrochemical noise (EN) data using MEM for pitting corrosion of 316 SS in chloride solution. Int. J. Electrochem. Sci. 2 (4), 301-310.

Sarmiento, E., González-Rodriguez, J.G., Uruchurtu, J., Sarmiento, O., Menchaca, M. (2009). Fractal analysis of the corrosion inhibition of carbon steel in a bromide solution by lithium chromate. Int. J. Electrochem. Sci. 4 (1), 144-155.

Shao, Y., Jia, C., Meng, G., Zhang, T., Wang, F. (2009). The role of a zinc phosphate pigment in the corrosion of scratched epoxy-coated steel. Corros. Sci. 51 (2), 371-379. http:// dx.doi.org/10.1016/j.corsci.2008.11.015.

Skerry, B.S., Eden, D.A. (1987). Electrochemical testing to assess corrosion protective coatings. Prog. Org. Coat. 15 (3), 269-285. http://dx.doi.org/10.1016/0033-0655(87)85015-X. 